\title{
CKS2 in human cancers: Clinical roles and current perspectives (Review)
}

\author{
HANYU YOU ${ }^{1 *}$, HUAYUE LIN ${ }^{1 *}$ and ZHONGYING ZHANG ${ }^{1,2}$ \\ ${ }^{1}$ Center for Clinical Laboratory, Xiamen University Affiliated Zhongshan Hospital, Xiamen, Fujian 361000; \\ ${ }^{2}$ State Key Laboratory of Molecular Vaccinology and Molecular Diagnostics, School of Public Health, \\ Xiamen University, Xiamen, Fujian 361005, P.R. China
}

Received November 16, 2014; Accepted January 23, 2015

DOI: $10.3892 / \mathrm{mco} .2015 .501$

\begin{abstract}
Cyclin-dependent kinase subunit 2 (CKS2) is indicated in the processes of cell cycle and cell proliferation. Through these processes, CKS 2 is identified as a cancer gene, but its role has not been well reviewed. The aim of the present study was to summarize the clinicopathological significance and the molecular mechanisms of CKS2 in human cancers. Its expression was upregulated in the majority of the types of cancer studied. CKS2 was shown to have a function in cancers of the digestive tract, genital tract, thyroid, nerve and certain other types of cancer. $C K S 2$ can promote progression of certain cancers via positive control of proliferation, invasion and migration. Downregulation of $C K S 2$ induces cancer cell apoptosis. CKS2 can change a multitude of cellular mechanisms in cancer pathogenesis by regulating the gene translation of numerous validated targets, such as $p 53, C D K 1$, cyclin A, cyclin $\mathrm{B} 1$, caspase- 3 and Bax. In addition, the molecular mechanism that causes aberrant expression of $C K S 2$ was epigenetic modification of $m i R-26 a$ and the Y-box-binding protein $1(Y B-1)$ gene. In conclusion, $C K S 2$ is commonly elevated in cancer, most likely due to its ability to promote cancer cell growth, invasion and migration through regulating certain significant genes. Understanding the mechanisms by which $C K S 2$ is involved with cancer pathogenesis will be useful in the development of tumor therapy for patients with cancer.
\end{abstract}

Correspondence to: Professor Zhongying Zhang, Center for Clinical Laboratory, Xiamen University Affiliated Zhongshan Hospital, 201-209 Hubin South Road, Siming, Xiamen, Fujian 361000, P.R. China

E-mail: zhangzy1121@xmu.edu.cn

${ }^{*}$ Contributed equally

Key words: CKS2, cancer, clinicopathological features, molecular mechanisms, biological function

\section{Contents}

1. Introduction

2. $C K S 2$ is highly expressed in malignant tumors

3. CKS2 expression correlates to clinicopathological features

4. Molecular mechanisms

5. CKS2 acts as a target gene

6. Conclusion

\section{Introduction}

The mammalian cyclin kinase subunit (CKS) family has two members; cyclin-dependent kinase subunit 1 (CKS1) and CKS2. CKS1 and CKS2 consist of 79 amino acids, and show $81 \%$ homology (1). Richardson et al (2) cloned the gene of human CKS for the first time in 1990; identifying human CKSI and $C K S 2$. Human $C K S 1$ is located in chromosome $8 \mathrm{q} 21$ and coding human CKS2 gene is located in chromosome 9q22 (3). Studies have shown that CKS1 and CKS2 proteins are associated with cell proliferation (4). Human CKS1 is required for $\mathrm{SCF}^{\text {Skp2 }}$-mediated ubiquitination and degradation of $\mathrm{P} 27^{\mathrm{kip} 1}$ that is essential for the $\mathrm{G}_{1} / \mathrm{S}$ transition during the cell cycle (5). Human CKS2 is essential for the first metaphase/anaphase transition of mammalian meiosis (6-8). The function of human CKS1 has been researched thoroughly, while the function of human CKS2 remains poorly understood. Increasing research is focusing on the association between human CKS2 and tumors. However, there is a lack of review on the clinical and functional roles of human CKS2 in human cancers. The present study is a review of the recent progress of human CKS2.

\section{CKS2 is highly expressed in malignant tumors}

Hepatocellular carcinoma. Shen et al (9) applied the technology of reverse transcription-quantitative polymerase chain reaction (RT-qPCR) and analyzed the mRNA level of $C K S 2$ in $\mathrm{HCC}$ and its adjacent liver tissues that either had cirrhosis or hepatitis, as well as in normal liver tissues. RT-qPCR revealed that the CKS2 mRNA level was significantly elevated in HCC. This study also used immunostaining analyses to determine the CKS2 protein expression in the tissues. High CKS2 protein expression was evident in 38 out of 48 HCC cases. 
Esophageal carcinoma. Our previous study investigated CKS2 in esophageal carcinoma (10). Wang et al (10) collected 26 paired esophageal carcinoma and its adjacent normal tissues. RT-qPCR revealed that in $65 \%$ of esophageal carcinoma tissues, the CKS2 mRNA level was highly expressed. Immunohistochemical staining analyses were performed to assess the protein levels of $C K S 2$ in 56 esophageal carcinoma tissues. The results showed that $61 \%$ of 56 cases had high CKS2 protein levels. Kita et al (11) also conducted studies of $C K S 2$ expression in esophageal squamous cell carcinoma. In the mRNA analysis, 93.5\% of patients (58/62) showed higher expression of CKS2 in cancerous compared to non-cancerous tissues.

Gastric cancer. Using RT-qPCR assays on eight human gastric cancer cell lines, Tanaka et al (12) observed the expression of $C K S 2$ mRNA. A significantly higher expression of $C K S 2$ mRNA was verified in the eight human gastric cancer cells compared to normal cells. RT-qPCR analysis of 109 paired clinical samples showed that 62 of 109 cases (56.9\%) exhibited higher levels of $C K S 2 \mathrm{mRNA}$ in the tumor tissues compared to the paired normal tissues.

Colorectal cancer (CRC). Notterman et al (13) applied semiquantitative RT-PCR reactions to compare $C K S 2$ expression levels between colon adenocarcinomas and paired normal colon tissues. The mean expression of $C K S 2$ in 18 cancerous tissues to normal tissues was $>4$-fold. Jung et al (14) verified that the CKS2 gene expression level was significantly enhanced in CRC tissues compared to normal tissues in 29 patient cases by RT-qPCR analysis. In another study of CKS2 in CRC, CKS2 was significantly augmented in the CRC tissues compared to the adjacent non-cancerous and normal colorectal tissues, using RT-qPCR analyses in combination with western blot (15). Li et al (16) also found that CKS2 was highly expressed in colon cancer.

Bladder cancer $(B C)$. Using microarray analysis, it was shown that $C K S 2$ expression was altered in BC. CKS2 was upregulated $>1.5$-fold in $\mathrm{BC}$ compared to normal bladder, and RT-qPCR verified the microarray results. Investigators identified that the $C K S 2$ gene showed a higher expression in BC $(2.1 \pm 0.64)$ compared to the normal bladder $(0.1 \pm 0.01)(17)$. Chen et al (18) measured the CKS2 mRNA level of 38 cases in superficial BC samples. The mean level, $2.4 \pm 0.2$, was equivalent to 10 times to normal samples $(0.2 \pm 0.3)$.

In other malignant tumors, such as glioma $(19,20)$, multiple myeloma (21), follicular thyroid carcinoma (22), prostate cancer (23), malignant melanoma (24), cervical cancer (25), laryngeal squamous cell carcinoma (26) and meningioma (27), CKS2 was also highly expressed.

\section{CKS2 expression correlates to clinicopathological features}

$C K S 2$ was upregulated in numerous malignant tumors and investigators have researched the association between $C K S 2$ expression and clinicopathological parameters.

Association between CKS2 expression with tumor size, histological tumor differentiation and pathological tumor-node-metastasis (pTNM) stage
Gastric cancer. Kang et al (28) performed immunohistochemical staining analyses and identified that $C K S 2$ was more strongly expressed in the less differentiated area compared to the well differentiated area, even in the same tumor tissue of each patient, occasionally. The study divided 60 cases of gastric cancer tissues into two groups; low and high CKS2 expression levels. Subsequently, the SPSS ${ }^{\circledR}$ software (SPSS, Inc., Chicago, IL, USA) package was used to determine the association between the results of the immunohistochemical study and the clinicopathological parameters. The analyses showed that a high level of $C K S 2$ strongly correlated with tumor size, histological tumor differentiation and pTNM stage. A total of $82.1 \%$ of the patients in the high CKS2 expression group had tumor diameters of $>4 \mathrm{~cm}$, whereas $73.3 \%$ were poorly differentiated and $83.3 \%$ were pTNM stage IV. Multivariate logistic analysis was used to assess the predictive value of the CKS2 expression status for clinicopathological significance. The results corroborated that histological tumor differentiation, pTNM stage and a high level of $C K S 2$ were significant covariables. A high $C K S 2$ expression level was also found to be closely associated with tumor size and pTNM stage in gastric cancer tissues (12).

Hepatocellular carcinoma. Shen et al (9) applied RT-qPCR and immunohistochemical staining analyses to determine the CKS2 mRNA and protein expression levels in 48 hepatocellular carcinoma tissues. The SPSS ${ }^{\oplus}$ statistical software package was used to study the associations between $C K S 2$ expression and various clinicopathological features. The analyses showed that a high $C K S 2$ expression level strongly correlated with poor differentiation. No association was found between $C K S 2$ overexpression and tumor size. In another study by Shen et al (29), the same results were exhibited in cholangiocarcinoma, and CKS2 overexpression was associated with poor differentiation.

$C R C$. Jung et al (14) studied the correlation between the $C K S 2$ gene expression level and clinical characteristics in CRC tissues. The analysis of variance statistical test was used and the results showed that a high level of $C K S 2$ expression was associated with early tumor stage. The CKS2 mRNA levels of 16 patients in stage II were higher compared to eight patients in stage III. Yu et al (15) also reported results that the overexpression of $C K S 2$ was correlated with the pathological stage in CRC tissues. Using western blot analyses, this study reported that tumor tissues from 15 stage III CRC patients demonstrated significantly higher CKS2 protein levels compared to those from 15 stage II patients. CKS2 expression was also correlated with tumor size and differentiation. The reason for the results differing from Jung et al (14) may be due to the smaller number of patients from stage III in the latter.

\section{CKS2 promotes cancer invasion and metastasis CKS2 promotes cancer invasion}

$B C$. In 2006, Kawakami et al (17) first reported that $C K S 2$ expression is strongly correlated with the progression of human BC. This study subjected $21 \mathrm{BC}$ samples to the RT-qPCR assay for $C K S 2$, and identified a considerable difference between superficial and invasive BC. CKS2 had a significantly greater level of upregulation in invasive compared to superficial $\mathrm{BC}(\mathrm{P}=0.04)$. 
Chen et al (18) used the RT-qPCR analysis on 45 patient samples with a diagnosis of superficial BC (of these, seven patients developed muscle-invasive BC during the follow-up period) and 10 samples from normal bladders. Expression of the CKS2 gene in superficial BC tissues was $>10$-fold higher compared to normal bladder tissues, and was $\sim 4$-fold higher following disease transition in patients developing muscle-invasive cancer. $C K S 2$ had significantly increased expression levels following transition from superficial $\mathrm{BC}$ to muscle-invasive cancer.

Gastric cancer. Tanaka et al (12) reported that 31 cases exhibited serosal invasion among 40 cases of high $C K S 2$ expression in gastric cancer tissues.

From these outcomes it can be inferred that $C K S 2$ may function as a tumor oncogene. $C K S 2$ may promote cancer invasion and it may be a useful biomarker for predicting disease outcome and the requirement for early preventive treatments.

CKS2 promotes lymph node metastasis. Lymph node metastasis is extremely common for cancers, and almost all will develop to lymph node metastasis of tumors (30).

Esophageal carcinoma. Kita et al (11) examined the mRNA level of $C K S 2$ in esophageal squamous cell carcinoma tissues from 62 esophageal cancer patients. The patients with values below the median expression level in tumor tissues were assigned to the low expression group, whereas those with values above the median were assigned to the high expression group. Of the 31 patients assigned to the high expression group, $26(83.9 \%)$ presented with lymphatic invasion. This result suggested that high expression of $C K S 2$ was significantly associated with the incidence of lymphatic invasion. Wang et al (10) also observed the correlation between high CKS 2 expression and lymph node metastasis in esophageal carcinoma tissues.

Gastric cancer. Tanaka et al (12) showed the results that 40 cases had high $C K S 2$ expression and 69 cases had low $C K S 2$ expression in 109 cases of gastric cancer tissues. Of the 40 cases with high $C K S 2$ expression, 32 (80\%) had lymph node metastasis, indicating that $C K S 2$ promotes lymph node metastasis. Kang et al (28) also observed the correlation between high $C K S 2$ expression and lymph node metastasis in gastric cancer tissues.

Cervical cancer. Lyng et al (31) identified the gene expression in 29 cervical cancer patients with and 19 patients without lymph node metastases using a genomic microarray technique. $C K S 2$ was noted to be elevated in node-positive compared to node-negative tumors.

From these outcomes it can be suggested that $C K S 2$ may function as a tumor oncogene and it promotes lymph node metastasis from carcinoma in situ.

CKS2 is associated with liver metastasis. The liver is the most common site of metastases from the digestive tract tumors. Tanaka et al (12) reported that five cases (12.5\%) had an incidence of liver metastasis in 40 gastric cancer tissues with a high level of CKS 2 mRNA expression. In 69 cases with a low level of $C K S 2$ mRNA expression, just one case (1.4\%) had the incidence of liver metastasis, indicating that high expression of CKS2 was associated with liver metastasis.

Lin et al (32) identified conflicting results to Tanaka et al (12). The mRNA profiling of $C K S 2$ was undertaken by RT-qPCR to confirm the differential expression between liver metastases and primary tumors. $C K S 2$ was noted to be downregulated in 28 metastases tissues relative to 40 primary colon tumors.

CKS2 is associated with the survival rate. The prognosis for cancer patients is extremely important. Tanaka et al (12) used Kaplan-Meier survival curves to evaluate the survival rate in gastric cancer patients according to the levels of $C K S 2$ mRNA expression. The overall 5-year survival rate was significantly higher in the $C K S 2$ low expression group (59.9\%) compared to the $C K S 2$ high expression group (23.9\%). Thus, CKS 2 mRNA expression was associated with the prognosis.

\section{Molecular mechanisms}

\section{CKS2 regulates the cell cycle}

CKS2 downregulates p53. CKS2 is a transcription target downregulated by p53 (7). Kang et al (28) used SUN638 and AGS gastric cancer cells that were transfected with GFP; GFP-CKS2 plasmids. The western blot results showed that the protein expression level of p53 in GFP-CKS2-overexpressing SUN638 and AGS cells was lower compared to the GFP control cells, suggesting that the increased CKS2 protein affected the protein level of p53. However, it requires further study to understand how $C K S 2$ overexpression causes the downregulation of $\mathrm{p} 53$.

CKS2 upregulates cyclin A, cyclin B1 and CDK1. Cholangiocarcinoma cells, QBC939, were stably transfected with shCKS2 or shCtrl plasmids, and stable CKS2-knockdown cells were established. RT-qPCR and western blot analysis were used to determine the cell cycle associated genes. $C K S 2$ downregulation resulted in a marked reduction in the protein and mRNA levels of cyclin A and cyclin B1 when compared with shCtrl cells. Flow cytometry showed that $C K S 2$ downregulation increased the cells at the $\mathrm{G}_{2} / \mathrm{M}$ phase and decreased the cells at the $\mathrm{S}$ and $\mathrm{G}_{1}$ phases. In this study, $C K S 2$-knockdown induced cholangiocarcinoma cell cycle arrest in $G_{2}$ phase by decreasing the expressions of cyclin A and cyclin B1. These results suggest that $C K S 2$ may serve as a cell cycle checkpoint protein for $\mathrm{S} / \mathrm{G}_{2}$ transition and this is one of the mechanisms of how $C K S 2$ promotes cholangiocarcinoma progression (29).

Tissues from 10 gastric cancer patients were prepared and RT-qPCR analysis was performed (28). Cell cycle regulators, cyclin $\mathrm{A}$, cyclin $\mathrm{B} 1$ and $C D K 1$, were evidently upregulated in tumor tissues compared to normal tissues. The study also used the small interfering RNA (siRNA)-mediated gene-silencing method to suppress the transcript level of $C K S 2$ in AGS gastric cancer cells. When the transcript levels of $C K S 1$ and $C D K 1$ were compared with the $C K S 2$ level, the genes were upregulated, possibly by a complicated mechanism in which the proteins that have high similarities, such as CKSs and CDKs, complement each other to maintain their basic role.

CDK1 and cyclin B1 are known to be important players in the cell cycle. Numerous studies have demonstrated that 
cyclin B1-CDK1 protein kinase, also known as mitosis promoting factor, is essential for mitosis and that in its absence, cells are unable to progress past the $\mathrm{G}_{2}$ phase of the cell cycle $(33,34)$. CKS2 binds to CDK1 via interaction with the catalytic subunit of CDK1, and subsequently affects cell cycle.

CKS2 regulates cell apoptosis. Tanaka et al (12) used the siRNA-mediated gene-silencing method to suppress the transcript level of $C K S 2$ in MKN74 gastric cancer cells and examined caspase- 3 expression by flow cytometry. CKS2-siRNA cells had increased caspase-3 activity (10.8\%) compared to the siRNA-negative control $(2 \%)$, indicating that the suppression of $C K S 2$ expression increased caspase-3 activity by $>5$-fold. The knockdown of $C K S 2$ expression increased Bax expression at the protein level, shown by western blot analyses. Caspase-3 and Bax proteins are involved in cell apoptosis, these results show that apoptosis is induced by inhibiting CKS2 expression.

Shen et al (29) observed the changes in the expression of Bcl-2 family proteins in cholangiocarcinoma cells by western blot analysis. Bax increased following CKS2 knockdown (shCKS2). The study also examined the activation of Bax by immunofluorescence and found that Bax activation increased in shCKS2 cells. In the nude mouse tumorigenesis experiment, the $32 \mathrm{kDa}$ proenzyme caspase- 3 was cleaved to obtain its active form of $17 \mathrm{kDa}$ following $C K S 2$ knockdown. These data suggest that $C K S 2$ knockdown enhances the susceptibility of cholangiocarcinoma cells to a Bax-mediated mitochondrial caspase-dependent apoptosis. To further confirm $C K S 2$ knockdown promoting cholangiocarcinoma cells under apoptosis, flow cytometry was used to evaluate the apoptotic susceptibility of QBC939-shCKS2 and shCtrl cells. Data indicated that the apoptosis rate of shCKS2 cells (42.5\%) was significantly higher compared to shCtrl cells (12.3\%) under hypoxia growth conditions. From these results it is shown that CKS2 downregulation can promote cholangiocarcinoma under apoptosis.

\section{CKS2 acts as a target gene}

CKS2 has its affect on the expression of downstream genes, mostly cyclin A, cyclin B1 and $C D K 1$. In certain studies, a variety of results indicated that $C K S 2$ is a direct target of specific genes.

CKS2 is a target of miR-26a. Lv et al (35) explored the $m i R$-26a-regulated target gene(s), using three publicly-available miRNA target prediction tools: TargetScan, ncRNA and mirecords. CKS2 was selected as a potential target.

To assess whether $m i R-26 a$ directly altered the expression of $C K S 2$, a fragment of the 3'untranslated region (UTR) of CKS2 mRNA [wild-type (wt) 3'UTR or the mutant sequence (mut 3'UTR)] containing the putative miR-26a binding sequence, was cloned into a luciferase reporter vector. HEK-293T cells were subsequently transfected with the wt or mut 3'UTR of CKS2 and miR-26a mimic. Luciferase expression was decreased by $\sim 50 \%$ when the wt 3'UTR and $m i R-26 a$ mimic were co-transfected, while the mut $3^{\prime} \mathrm{UTR}$ had no effect on luciferase activity.
This study also verified that $C K S 2 \mathrm{mRNA}$ and protein levels were decreased by $m i R$-26a overexpression in TPC-1 and CGTH W 3 cells, and this effect was attenuated by anti-miR-26a. These results indicate that $C K S 2$ is a direct target of $m i R-26 a$, and $m i R-26 a$ modulates cell growth and tumorigenesis of papillary thyroid carcinoma via regulating $C K S 2$.

Y-box-binding protein 1 (YB-1) gene affects CKS2 expression. YB-1 belongs to a family of RNA-and DNA-binding proteins. It performs pleiotropic cellular functions, including transcriptional regulation, translational regulation, DNA repair and drug resistance (36). To have an improved insight into the role of YB-1 on cell proliferation, Yu et al (37) used T-47D breast cancer cells that were transfected with $Y B-1$ siRNA. The $C K S 2$ gene was downregulated in response to siYB-1 treatment by RT-qPCR analysis; however, the investigators did not discuss the interaction between $Y B-1$ and $C K S 2$. There may be a link, and silencing of the $Y B-1$ gene may induce $C K S 2$ downregulation, but more experiments are required to clarify the regulation.

\section{Conclusion}

A number of human malignancies are characterized by $C K S 2$ overexpression, and it is generally known as an oncogene. The role of the CKS2 protein in tumors has attracted increasing interest. In recent years, investigators have explored the gene function of $C K S 2$ by gene knockout or overexpression studies. CKS2 has abnormal expression in a variety of malignant tumor tissues and was closely associated with certain biological behavior, such as tumor development, progression and metastasis. Certain investigators have conducted a more in-depth research of $C K S 2$, suggesting that $C K S 2$ can increase the cell cycle proteins, cyclin A, cyclin B1 and CDK1, thus promoting cancer cell proliferation. $C K S 2$ was closely associated with cell apoptosis through a Bax-mediated mitochondrial caspase-dependent apoptosis. Simultaneously, $C K S 2$ was also regulated by $m i R-26 a$ and the $Y B-1$ gene. The present review allows an unobstructed view on the clinicopathological significance and the molecular mechanisms of CKS2 in human cancers. The application is promising in cancer due to the significant role of the CKS2 protein. Investigating the association between $C K S 2$ and tumors, cell cycle and apoptosis depends on the further studies of the CKS2 biological functions. Along with the development of associated experimental studies, the CKS2 protein could become a new biomarker of tumors for tumor therapy.

\section{Acknowledgements}

This study was supported by the National Nature Science Foundation of China (grant no. 81311120473).

\section{References}

1. Williams RT, Wu L, Carbonaro-Hall DA, Tolo VT and Hall FL: Identification of a novel cyclin-like protein in human tumor cells. J Biol Chem 268: 8871-8880, 1993.

2. Richardson HE, Stueland CS, Thomas J, Russell P and Reed SI: Human cDNAs encoding homologs of the small p34Cdc28/Cdc2-associated protein of Saccharomyces cerevisiae and Schizosaccharomyces pombe. Genes Dev 4: 1332-1344, 1990. 
3. Demetrick DJ, Zhang H and Beach DH: Chromosomal mapping of the human genes CKS1 to $8 \mathrm{q} 21$ and CKS2 to 9q22. Cytogenet Cell Genet 73: 250-254, 1996.

4. Urbanowicz-Kachnowicz I, Baghdassarian N, Nakache C, Gracia D, Mekki Y, Bryon PA and Ffrench M: ckshs expression is linked to cell proliferation in normal and malignant human lymphoid cells. Int J Cancer 82: 98-104, 1999.

5. Ganoth D, Bornstein G, Ko TK, Larsen B, Tyers M, Pagano M and Hershko A: The cell-cycle regulatory protein Cks1 is required for $\mathrm{SCF}(\mathrm{Skp} 2)$-mediated ubiquitinylation of $\mathrm{p} 27$. Nat Cell Biol 3: 321-324, 2001.

6. Spruck CH, de Miguel MP, Smith AP, Ryan A, Stein P, Schultz RM, Lincoln AJ, Donovan PJ and Reed SI: Requirement of Cks2 for the first metaphase/anaphase transition of mammalian meiosis. Science 300: 647-650, 2003.

7. Rother K, Dengl M, Lorenz J, Tschöp K, Kirschner R, Mössner J and Engeland K: Gene expression of cyclin-dependent kinase subunit Cks2 is repressed by the tumor suppressor p53 but not by the related proteins p63 or p73. FEBS Lett 581: 1166-1172, 2007.

8. Martinsson-Ahlzén H-S, Liberal V, Grünenfelder B, Chaves SR Spruck CH and Reed SI: Cyclin-dependent kinase-associated proteins $\mathrm{Cks} 1$ and $\mathrm{Cks} 2$ are essential during early embryogenesis and for cell cycle progression in somatic cells. Mol Cell Biol 28 5698-5709, 2008.

9. Shen DY, Fang ZX, You P, Liu PG, Wang F, Huang CL, Yao XB, Chen ZX and Zhang ZY: Clinical significance and expression of cyclin kinase subunits 1 and 2 in hepatocellular carcinoma. Liver Int 30: 119-125, 2010

10. Wang JJ, Fang ZX, Ye HM, You P, Cai MJ, Duan HB Wang $F$ and Zhang ZY: Clinical significance of overexpressed cyclin-dependent kinase subunits 1 and 2 in esophageal carcinoma. Dis Esophagus 26: 729-736, 2013.

11. Kita Y, Nishizono Y, Okumura $\mathrm{H}$, et al: Clinical and biological impact of cyclin-dependent kinase subunit 2 in esophageal squamous cell carcinoma. Oncol Rep 31: 1986-1992, 2014.

12. Tanaka F, Matsuzaki S, Mimori K, Kita Y, Inoue H and Mori M: Clinicopathological and biological significance of CDC28 protein kinase regulatory subunit 2 overexpression in human gastric cancer. Int J Oncol 39: 361-372, 2011.

13. Notterman DA, Alon U, Sierk AJ and Levine AJ: Transcriptional gene expression profiles of colorectal adenoma, adenocarcinoma, and normal tissue examined by oligonucleotide arrays. Cancer Res 61: 3124-3130, 2001

14. Jung Y, Lee S, Choi HS, et al: Clinical validation of colorectal cancer biomarkers identified from bioinformatics analysis of public expression data. Clin Cancer Res 17: 700-709, 2011.

15. Yu M,Zhong M and Qiao Z: Expression and clinical significance of cyclin kinase subunit 2 in colorectal cancer. Oncol Lett 6 : 777-780, 2013

16. Li M, Lin Y-M, Hasegawa S, et al: Genes associated with liver metastasis of colon cancer, identified by genome-wide cDNA microarray. Int J Oncol 24: 305-312, 2004.

17. Kawakami K, Enokida H, Tachiwada T, Gotanda T, Tsuneyoshi K, Kubo H, Nishiyama K, Takiguchi M, Nakagawa M and Seki N: Identification of differentially expressed genes in human bladder cancer through genome-wide gene expression profiling. Oncol Rep 16: 521-531, 2006.

18. Chen R, Feng C and Xu Y: Cyclin-dependent kinase-associated protein Cks2 is associated with bladder cancer progression. J Int Med Res 39: 533-540, 2011

19. Scrideli CA, Carlotti CG Jr, Okamoto OK, et al: Gene expression profile analysis of primary glioblastomas and non-neoplastic brain tissue: Identification of potential target genes by oligonucleotide microarray and real-time quantitative PCR. J Neurooncol 88: 281-291, 2008

20. Rickman DS, Bobek MP, Misek DE, Kuick R, Blaivas M, Kurnit DM, Taylor J and Hanash SM: Distinctive molecular profiles of high-grade and low-grade gliomas based on oligonucleotide microarray analysis. Cancer Res 61: 6885-6891, 2001.
21. Haaber J, Abildgaard N, Knudsen LM, Dahl IM, Lodahl M, Thomassen M, Kerndrup GB and Rasmussen T: Myeloma cell expression of 10 candidate genes for osteolytic bone disease. Only overexpression of DKK1 correlates with clinical bone involvement at diagnosis. Br J Haematol 140: 25-35, 2008.

22. Williams MD, Zhang L, Elliott DD, Perrier ND, Lozano G, Clayman GL and El-Naggar AK: Differential gene expression profiling of aggressive and nonaggressive follicular carcinomas. Hum Pathol 42: 1213-1220, 2011

23. Lan Y, Zhang Y, Wang J, Lin C, Ittmann MM and Wang F: Aberrant expression of Cks1 and Cks2 contributes to prostate tumorigenesis by promoting proliferation and inhibiting programmed cell death. Int J Cancer 123: 543-551, 2008.

24. de Wit NJ, Rijntjes J, Diepstra JH, van Kuppevelt TH, Weidle UH, Ruiter DJ and van Muijen GN: Analysis of differential gene expression in human melanocytic tumour lesions by custom made oligonucleotide arrays. Br J Cancer 92: 2249-2261, 2005.

25. Wong YF, Cheung TH, Tsao GS, et al: Genome-wide gene expression profiling of cervical cancer in Hong Kong women by oligonucleotide microarray. Int J Cancer 118: 2461-2469, 2006.

26. Ni RS, Shen X, Qian X, Yu C, Wu H and Gao X: Detection of differentially expressed genes and association with clinicopathological features in laryngeal squamous cell carcinoma. Oncol Lett 4: 1354-1360, 2012.

27. Menghi F, Orzan FN, Eoli M, et al: DNA microarray analysis identifies CKS2 and LEPR as potential markers of meningioma recurrence. Oncologist 16: 1440-1450, 2011

28. Kang MA, Kim JT, Kim JH, Kim SY, Kim YH, Yeom YI, Lee Y and Lee HG: Upregulation of the cycline kinase subunit CKS2 increases cell proliferation rate in gastric cancer. J Cancer Res Clin Oncol 135: 761-769, 2009.

29. Shen DY, Zhan YH, Wang QM, Rui G and Zhang ZM: Oncogenic potential of cyclin kinase subunit-2 in cholangiocarcinoma. Liver Int 33: 137-148, 2013.

30. Gendreau KM and Whalen GF: What can we learn from the phenomenon of preferential lymph node metastasis in carcinoma? J Surg Oncol 70: 199-204, 1999.

31. Lyng H, Brøvig RS, Svendsrud DH, Holm R, Kaalhus O, Knutstad K, Oksefjell H, Sundfør K, Kristensen GB and Stokke T: Gene expressions and copy numbers associated with metastatic phenotypes of uterine cervical cancer. BMC Genomics 7: 268 , 2006.

32. Lin HM, Chatterjee A, Lin YH, Anjomshoaa A, Fukuzawa R, McCall JL and Reeve AE: Genome wide expression profiling identifies genes associated with colorectal liver metastasis. Oncol Rep 17: 1541-1549, 2007.

33. Dorée M and Hunt T: From Cdc2 to Cdk1: when did the cell cycle kinase join its cyclin partner? J Cell Sci 115: 2461-2464, 2002.

34. Vassilev LT, Tovar C, Chen S, Knezevic D, Zhao X, Sun H, Heimbrook DC and Chen L: Selective small-molecule inhibitor reveals critical mitotic functions of human CDK1. Proc Natl Acad Sci USA 103: 10660-10665, 2006.

35. Lv M, Zhang X, Li M, Chen Q, Ye M, Liang W, Ding L, Cai H, Fu D and Lv Z: miR-26a and its target CKS2 modulate cell growth and tumorigenesis of papillary thyroid carcinoma. PLoS ONE 8: e67591, 2013.

36. Kohno K, Izumi H, Uchiumi T, Ashizuka M and Kuwano M The pleiotropic functions of the Y-box-binding protein, YB-1. BioEssays 25: 691-698, 2003.

37. Yu YN, Yip GW, Tan PH, Thike AA, Matsumoto K, Tsujimoto M and Bay BH: Y-box binding protein 1 is up-regulated in proliferative breast cancer and its inhibition deregulates the cell cycle. Int J Oncol 37: 483-492, 2010. 